\title{
Rethinking Community Benefit Programs-A New Vision for Hospital Investment in Community Health
}

\author{
Pooja Chandrashekar, $A B^{7}$, Rebekah Gee, $M D, M P H^{2}$, and Jay Bhatt, DO, MPH, MPA ${ }^{3}$ \\ ${ }^{1}$ Harvard Medical School, 25 Shattuck Street, Boston, MA, USA; '2Louisiana State University School of Medicine, New Orleans, USA; ${ }^{3}$ University of Illinois \\ Chicago School of Public Health, Chicago, USA.
}

$\mathrm{J}$ Gen Intern Med 37(5):1278-80

DOI: $10.1007 / \mathrm{s} 11606-021-07324-0$

(c) The Author(s) under exclusive licence to Society of General Internal Medicine 2021

$\mathrm{O}$ ver the past decade, hospitals have been tasked with a growing responsibility to address the social determinants of health. One promising avenue for investment is the "community benefit" that nonprofit hospitals must provide in exchange for their tax-exempt status. However, there is significant debate around whether community benefits actually serve their purpose in improving community health.

Over 58\% of US hospitals are nonprofit, and historically, these hospitals have tended to provide community benefit through charity care - free or discounted care for low-incomepatients - rather than making investments in social services or community organizations. One study showed that nonprofit hospitals spent over $80 \%$ of their community benefit spending on charity care and payment shortfall from Medicaid, compared to just $12 \%$ on community health. ${ }^{1}$

There are several reasons for this discrepancy. First, strengthening the healthcare safety net is an important community need. Second, it is easier for hospitals to continue investing in clinical programs rather than building the infrastructure needed to address social determinants. Finally, hospital accounting systems are designed to better track clinical spending, making it difficult to measure the impact of community health initiatives. ${ }^{2}$

Though charity care is critical, especially in communities with high levels of medical debt, a broader perspective is needed. Clinical care is only one of many activities that influence community health and policy reforms such as the Affordable Care Act (ACA) have expanded access to health insurance, reducing the need for uncompensated care and creating a compelling (albeit unrealized) opportunity for hospitals to invest these gains back into the communities they serve. $^{3}$

Received May 25, 2021

Accepted December 13, 2021

Published online January 19, 2022

\section{THE WAY FORWARD}

Broadly speaking, there are two ways hospitals can use community benefits as a lever to improve community health: first, by investing in programs that identify and address patient's social needs within the hospital, and second, by investing in community-based programs.

Understandably, most hospitals have focused on the first strategy, such as facilitating access to medical interpreters and screening patients for social needs. However, these do not tackle the root causes of poor health (e.g., poverty, food insecurity, housing instability, access to education). Moreover, in-hospital programs are rarely designed in concert with the community and often lack follow-up once patients leave the hospital. ${ }^{4}$

In this Viewpoint, we advocate for a greater focus on community-based investment. Some argue that hospitals are not the right vehicle for community health investments and should concentrate their efforts on improving care within the hospital. However, in many ways, hospitals are the ideal vehicle. Hospitals have a deep understanding of how patients' social needs impact their clinical conditions and, as anchor institutions in many communities, the financial and human capital to change conditions. Moreover, they often have an existing network of community partners they can readily tap into. ${ }^{4}$ And while nonprofit hospitals vary in their financial bandwidth, many do have the resources to make communitydirectedinvestments - in fact, seven of the ten most profitable US hospitals are nonprofit, each earning over $\$ 160$ million in annual profit, and altogether nonprofit hospitals provided $\$ 105$ billion in community benefits in 2018 .

Community benefit programs can be a powerful tool for hospitals to make community-based investments. This requires (1) policy reforms that incentivize investments in community health, (2)hospital-community partnerships, and (3) a renewed focus on the "investment" in community investment.

Policy Reforms That Incentivize Community Investment. The ACA mandated that nonprofit hospitals conduct a community health needs assessment (CHNA) every 3 years, document and report their findings, and develop an implementation strategy. Despite these requirements, the proportion of hospitals' community benefit spending on evidence-driven community-based activities has not increased. ${ }^{3}$ There is a need 
for more substantial policy reform around what qualifies as a community benefit, how community benefit spending should be allocated, and rules around reporting and oversight.

The IRS draws a distinction between community health improvement activities and "community-building" activities. This means that community health improvement activities, such as diabetes education or summer programs to expose youth to medicine, often qualify as community benefits, while "community-building" activities, such as economic development and affordable housing, might not count unless hospitals submit additional documentation demonstrating their impact on health (which can be challenging to quantify). ${ }^{5}$

One possible solution is for the IRS to allow communitybuilding activities to count towards hospitals' total community benefit spending. If these activities address community health needs, hospitals should not be required to report community health improvement and community-building activities separately.

In addition to the ambiguity around which activities qualify as community benefit, there is no minimum standard for what percentage of community benefit spending should go towards community-directed investments. Some states, such as Oregon, are establishing a required minimum for overall community benefit spending, but we think this needs to go a step further and require hospitals to commit a set percentage of their community benefit spending towards communitydirected investments. ${ }^{6}$

Given that hospitals in low-income areas have less flexibility and resources to spend on community programs compared to hospitals in affluent areas, a minimum standard for community-directed spending should be unique to each hospital based on their revenues and clinical expenditures. Allowing hospitals in the same geographic region to pool community benefit spending can also even out differences in resource availability.

These reforms should be accompanied by more stringent regulations around reporting. Currently, the IRS tracks community benefit spending through hospitals' Form 990s, but a study by Modern Healthcare found significant inconsistencies and errors in the section used to document community benefit spending. ${ }^{7}$ This can make it difficult to trace where community benefit dollars go.

Such regulations are perhaps best implemented at the state level given a greater understanding of the community being served. For example, Connecticut hospitals are required to submit a public document to the state's Office of Health Strategy detailing how their community benefit expenditures address community needs. ${ }^{6}$ Local oversight is useful in assessing whether investments are appropriate and sufficient.

Hospital-Community Partnerships. Community benefit programs can bridge the disconnect between a hospital's mission and a community's needs. Though the ACA mandated that hospitals conduct CHNAs in collaboration with community members, hospitals are not required to involve community partners in their implementation strategy.

Building effective hospital-community partnerships begins with setting clear expectations. For example, when partnering with a local food bank to distribute healthy food in neighborhoods with high rates of food insecurity, Indiana University Health created a memorandum of understanding to establish mutual understanding of expectations, roles, and measurable outcomes. ${ }^{8}$ Hospitals and community groups often have different, sometimes competing, priorities and cultures, making it critical to spend time upfront building consensus.

The next step is structuring the partnership in a way that reflects the genuine voice of each partner. These structures can vary-a series of interviews with hospital and community leaders revealed partnerships range from informal (e.g., ad hoc task forces, broad alliances) to more defined (e.g., steering committees, advisory boards) to formal (e.g., creation of a joint venture). ${ }^{8}$ Regardless of the specific structure, successful partnerships enabled information-sharing between hospitals and community groups, regular meetings to discuss progress, and iterative adjustments.

In addition, effective partnerships facilitate buy-in from community organizations. A good example is PIH Health, which partnered with the Los Nietos School District to form Healthy Los Nietos, a school-based collaborative to increase the number of students maintaining a healthy weight. In addition, the hospital invested resources, financial and otherwise, into the school district and helped raise awareness in the community around the district's programs. ${ }^{8}$ Ensuring all partners benefit is key to building a sustainable relationship.

Community benefit laws could require hospitals to complete each of these steps - a memorandum of understanding, creating an effective partnership structure, and buy-in from community partners - as part of their implementation strategy.

\section{Bring Back the "Investment" of Community Investment.} While banks remain at the forefront of lending, there is an opportunity for nonprofit hospitals with adequate financial resources to lend a portion of their profits at low interest rates to community groups or small businesses. The amount they lend could qualify as community benefits.

This idea is especially relevant in communities that have suffered the impacts of structural racism and damaging policies. For example, Boston Medical Center has committed a $\$ 600,000$ zero-percent loan to fund an affordable housing development in Roxbury, a historically under-resourced and disinvested neighborhood. The loan will be paid back over 20 years. ${ }^{9}$

In communities where hospitals lack the capital necessary to make investments on their own, they could leverage community development financial institutions (CDFI). CDFIs borrow funds from banks, corporations, philanthropies, and government agencies and lend this capital to community partners to facilitate projects. Recently, the American Hospital 
Association partnered with the Local Initiatives Support Corporation, a CDFI, to raise capital to support ten hospitals for projects ranging from improved housing to opening grocery stores.

These changes are not easy to implement. Addressing patients' social needs within the hospital is important, but it is time for hospitals to also invest in the community outside their walls. Doing so requires forging new partnerships, policy reform, and, most importantly, challenging the centuries-old assumption that the mission of hospitals is solely clinical care.

Corresponding Author: Pooja Chandrashekar, AB; Harvard Medical School, 25 Shattuck Street, Boston, MA 02115, USA (e-mail: pooja chandrashekar@hms.harvard.edu).

\section{Declarations:}

Conflict of Interest: We have no conflicts of interest to note.

\section{REFERENCES}

1. Chaiyachati KH, Qi M, Werner RM.Non-profit hospital community benefit spending based on local sociodemographics. J Health Care Poor Underserved. 2018;29(4):1259-1268. https://doi.org/10.1353/hpu.2018. 0093. PMID: 30449744; PMCID: PMC6425942.
2. Rozier M, Goold S, Singh S. How Should Nonprofit Hospitals' Community Benefit Be More Responsive to Health Disparities? AMA J Ethics. 2019 Mar 1;21(3):E273-280. https://doi.org/10.1001/amajethics.2019.273.

3. Kanter GP, Nabet B, Matone M, Rubin DM. Association of State Medicaid Expansion With Hospital Community Benefit Spending. JAMA Netw Open. 2020 May 1;3(5):e205529. https://doi.org/10.1001/jamanetworkopen. 2020.5529. PMID: 32469411; PMCID: PMC7260619.

4. "Encouraging Nonprofit Hospitals To Invest In Community Building: The Role Of IRS 'Safe Harbors', Health Affairs Blog, February 11, 2014. https://doi.org/10.1377/hblog20140211.037060.

5. Bakken E, Kindig D, Boufford J. What "Community Building" Activities are Nonprofit Hospitals Reporting as Community Benefit?. Front Public Health Serv Syst Res 2014;3(5):e10. https://doi.org/10.13023/FPHSSR. 0305.01.

6. Clary A, Higgins E. Oregon and Connecticut Hold Hospitals Accountable for Meaningful Community Benefit Investment. National Academy for State Health Policy. August 29, 2019. Accessed May 5, 2021. https://www. nashp.org/oregon-and-connecticut-hold-hospitals-accountable-for-meaningful-community-benefit-investment/.

7. Kacik A. InDepth: Flaws in hospital community benefit reporting create knowledge vacuum. Modern Healthcare. December 1, 2018. Accessed April 27, 2021. https://www.modernhealthcare.com/article/20181201/ NEWS/181119965/indepth-flaws-in-hospital-community-benefitreporting-create-knowledge-vacuum.

8. Creating effective hospital-community partnerships to build a Culture of Health. Health Research \& Educational Trust. August 2016. Accessed on September 27, 2021. https://www.aha.org/system/files/hpoe/ReportsHPOE/2016/creating-effective-hospital-community-partnerships.pdf.

9. Gaskins ASP, Steinitz R, Hacke R.Addressing the Social Determinants of Health. Center for Community Investment. November 2020. Accessed on October 7, 2021. https://centerforcommunityinvestment.org/sites/default/ files/Adressing\%20the\%20Social\%20Determinants\%20of\%2OHealth.pdf.

Publisher's Note Springer Nature remains neutral with regard to jurisdictional claims in published maps and institutional affiliations. 